\title{
The merits of plasmonic desalination
}

To the Editor - Zhou et al. described ${ }^{1}$ a novel plasmonic device that may be a good broadband solar absorber, but the assertion of it offering a new efficient method for desalination is problematic as the reported efficiency is more than two orders of magnitude inferior to that of off-the-shelf, affordable desalination systems in general, and vastly below even specifically solardriven desalination systems in particular.

The desalination rate reported by Zhou and colleagues of $5.7 \mathrm{~kg} \mathrm{~h}^{-1} \mathrm{~m}^{-2}$ for seawater at a solar irradiance of $4 \mathrm{~kW} \mathrm{~m}^{-2}$ is equivalent to a specific energy consumption (SEC) of $702 \mathrm{kWh} \mathrm{m}^{-3}$. The SEC comprises a dominant - albeit not exclusive - factor in both economic and feasibility evaluations ${ }^{2}$. (It is misleading to claim that solar input is 'free', because of the substantial capital cost of all solar collection, conversion and collateral elements.)

The thermodynamic limit for desalting seawater (salt concentration of $\sim 35 \mathrm{~g} \mathrm{~kg}^{-1}$, at a temperature of $300 \mathrm{~K}$ ) is $0.76 \mathrm{kWh} \mathrm{m}^{-3}$, although it can only be realized with workdriven processes such as reverse osmosis, and only in the reversible limit of zero flux $^{2}$. The SEC reported for commercial reverse osmosis desalination plants - with pragmatic recovery ratios of $\sim 35-50 \%$ (recovery ratio denoting desalted water generation relative to seawater input) - is now below $3 \mathrm{kWh} \mathrm{m}^{-3}$, which is more than two orders of magnitude superior to the solar desalination results reported by Zhou and colleagues.
The second law of thermodynamics mandates an inherently higher SEC for thermal-driven desalination ${ }^{2}$. Indeed, available waste-heat-recovery desalination technologies at $90^{\circ} \mathrm{C}$ have achieved an SEC of $190 \mathrm{kWh} \mathrm{m}^{-3}$ (ref. 2), which is still a factor of 3.7 better than the solar desalination system described by Zhou and colleagues. (Thermal desalination plants commonly operate at a recovery ratio of $\sim 35 \%$.)

Confusion may stem from Zhou and colleagues' system being shown to efficiently deliver the heat of vaporization that, by itself, is equivalent to an SEC of $\sim 600 \mathrm{kWh} \mathrm{m}^{-3}$. (Desalination predominantly relates to a mass-transfer process with the associated change in chemical potential, and does not imply vaporization, even if vaporization is used in some thermal desalination procedures.) The fact that thermal desalination plants have achieved SEC values far below $600 \mathrm{kWh} \mathrm{m}^{-3}$ derives from effective heat regeneration via the use of multiple effects before any remaining unutilized heat is ultimately rejected to the environment ${ }^{2}$.

To sharpen the issue to solar-driven desalination (not necessarily thermal), consider a photovoltaic-driven reverse osmosis system. With off-the-shelf, affordable photovoltaic systems, net conversion efficiencies to alternating current electricity are now at $\sim 20 \%$, with net SEC values of $\sim 15 \mathrm{kWh} \mathrm{m}^{-3}$ achievable, that is, about a factor of 47 more efficient than the solar desalination system of Zhou and colleagues.

Even if one mandates solar thermal input - replacing the waste-heat recovery noted above with input temperatures up to $\sim 90^{\circ} \mathrm{C}-$ then with the thermal conversion efficiency of existing stationary solar collectors exceeding $60 \%$, the net SEC can be $\sim 350 \mathrm{kWh} \mathrm{m}^{-3}$, that is, a factor of 2 better than Zhou and colleagues' solar desalination system.

There is certainly room for improvement in the economics and performance of today's commercial desalination plants ${ }^{2}$. While the idea presented by Zhou et al. might appear intriguing, the fact that its SEC values are inordinately higher than those of existing commercial plants would appear to render the notion as questionable for efficient desalination, even at the proof-of-concept level.

References

1. Zhou, L. et al. Nat. Photon. 10, 393-399 (2016).

2. Gordon, J. M. \& Chua, H. T. Desalination 386, 13-18 (2016).

Jeffrey M. Gordon ${ }^{1 \star}$ and Hui Tong Chua ${ }^{2 \star}$

'Department of Solar Energy and

Environmental Physics, Jacob Blaustein

Institutes for Desert Research, Ben-Gurion

University of the Negev, Sede Boqer Campus

84990, Israel. ${ }^{2}$ School of Mechanical and

Chemical Engineering, The University of

Western Australia, 35 Stirling Highway, Perth,

Western Australia 6009, Australia.

*e-mail: jeff@bgu.ac.il; huitong.chua@uwa.edu.au

\section{Reply to 'The merits of plasmonic desalination'}

Zhou et al. reply - We would like to thank Gordon and Chua for their remarks on our Letter ${ }^{1}$, and hope that this dialogue will help to further clarify the differences and applications of various desalination technologies.

We reported a plasmon-enhanced solar desalination device equipped with an $\mathrm{Al}$ nanoparticle plasmonic absorber to enable efficient and effective desalination performance. We made it very clear in our Letter that the aim was to provide a complementary portable or personalized solar desalination solution ${ }^{1,2}$, particularly needed in developing countries and remote areas without infrastructures.
In their Correspondence ${ }^{3}$, Gordon and Chua did not question any scientific points in the paper, but focused almost solely on the specific energy consumption (SEC) value. For almost all well-developed centralized desalination solutions (such as reverse osmosis, multi-effect distillation, multistage flash, and so on) for which the energy consumption constitutes the principal cost, it is well known that the SEC is certainly one of the most important factors. However, for personalized or portable desalination strategies, other factors such as water production rate, energy transfer efficiency, water quality as well as degree of portability and convenience are just as important, if not more so. As evidence, the daily yield of water and energy transfer efficiency are the two factors generally used in the field of solar stills $s^{4,5}$. After the publication of our work, the feedback we received from across the world also confirmed that when people do not have access to adequate funds and stable power to build and run a centralized desalination plant, the SEC value is not their primary concern. In the end, what really matters to them is how easily they can build the portable device and the clean water production rate compared with its footprint. The unique interfacial solar steam generation mechanism with high energy transfer efficiency we demonstrated can enable an 